\title{
Impact of the SARS-CoV-2 pandemic on cystic fibrosis centres and care: survey results from US centres
}

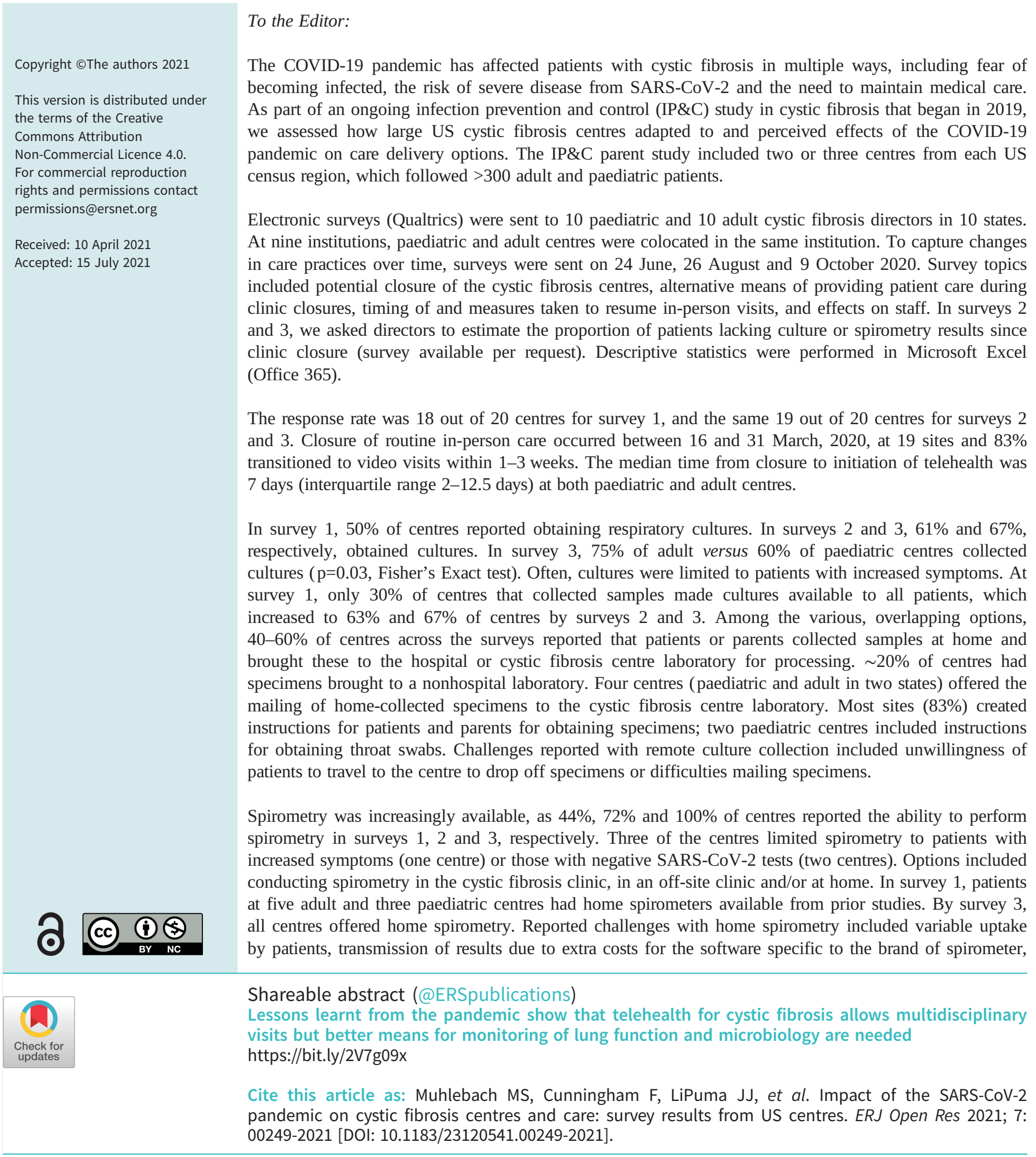


and, especially for children, concerns about reliability of results due to lack of coaching during the manoeuvres. Changes to spirometry in clinic included portable spirometers in the examination room and spirometry in the pulmonary function laboratory with newly installed high-efficiency particulate-absorbing filters. Two sites had pre-existing negative pressure in their pulmonary function laboratories. Healthcare personnel at all centres wore gloves, gowns, eye protection and masks (eight sites, only N95 respirators; eight sites, only surgical masks; three sites, either) while performing spirometry.

Most sites reopened in May and June 2020 (range 15 April to 14 September, 2020). By survey 3, 84\% (16 out of 19 centres) had reopened for in-person visits. In open-ended questions, several sites reported special IP\&C requirements (e.g. no waiting room and closing examination rooms for an hour between patients). We inquired about estimated proportion of patients lacking in-person visits, spirometry and/or cultures. This proportion decreased between surveys 2 and 3, but two adult centres estimated no changes over time. Figure 1 shows survey 3 responses.

Lay-offs occurred at two (11\%) centres and furloughs occurred over time in 16-32\% of centres, but hiring freezes were reported by $74 \%, 63 \%$ and $53 \%$ of centres in surveys 1, 2 and 3, respectively. Open comments from the sites mentioned that cystic fibrosis team members (respiratory therapists, dieticians and social workers) were temporarily reassigned to other hospital areas and/or conducted visits remotely from home. The cystic fibrosis centre space was reassigned temporarily at one centre.

Our surveys of geographically dispersed US cystic fibrosis centres showed nearly simultaneous closure to routine clinical care but variable reopening dates. Presumably, the rapid start of the pandemic stimulated consensus across the country, while regional factors influenced decisions to reopen for routine care. Of note, a limitation is generalisability, as the study included only large cystic fibrosis centres and smaller centres may have had different experiences. The majority of centres retained all their staff and transitioned rapidly to telehealth. Thus, patients were able to maintain urgently needed multidisciplinary care. However, performing cystic fibrosis-specific monitoring (spirometry and culture) was more challenging, with a gap between availability and reported performance. Ability to obtain spirometry was enhanced by availability of commercial systems and provision of home spirometers by the Cystic Fibrosis Foundation. Yet, the proportion of patients actually performing spirometry was affected by extrapandemic related factors and could not be reliably measured. Training and education for home spirometry had to be performed remotely, no comparative values to clinic spirometry were available and the costs of additional software was not supported by all cystic fibrosis centres, leading to delays in data transmission. As noted in prior studies, uptake of the home monitoring varied between patients [1].

Obtaining cultures proved more problematic than obtaining spirometry; several centres still reported lack of cultures 7 months into the pandemic (figure 1). The lack of cultures is especially concerning in patients without chronic infections who benefit from early detection and treatment of Pseudomonas aeruginosa [2]. Potentially, even collected specimens were suboptimal due to delays in shipping of specimens, and because processing of cystic fibrosis respiratory cultures requires special expertise and family members may be uncomfortable obtaining throat swabs. Furthermore, the pandemic began 4 months after approval and widespread prescription of highly effective triple cystic fibrosis transmembrane conductance regulator modulators, which probably reduced the number of patients able to produce sputum, further reducing culture rates among teenagers and adults. In contrast, fewer healthcare encounters and enhanced attention to IP\&C during the pandemic might decrease the number of new infections. The effect of reduced
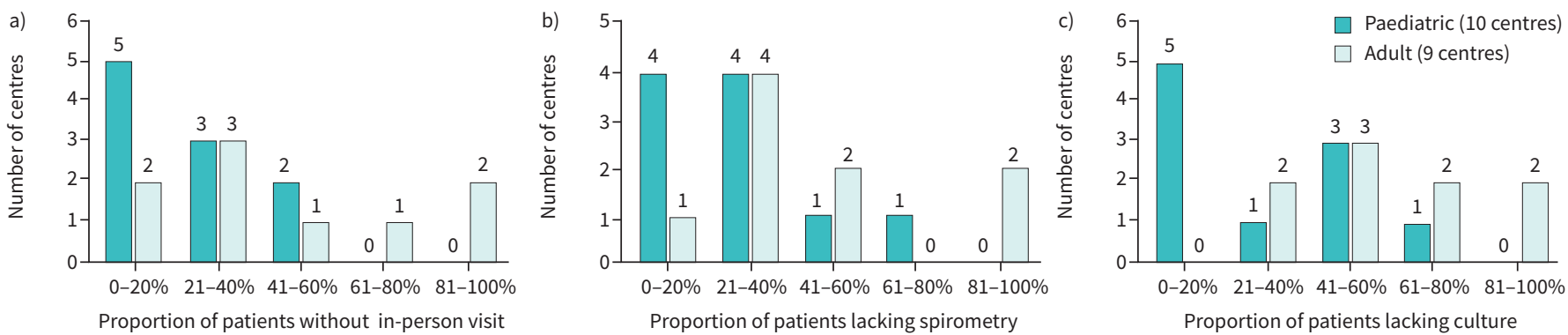

FIGURE 1 Each panel shows the number of centres who estimated the given proportion of patients still lacking a) in-person visits, b) spirometry and c) respiratory culture at the time of survey 3 . The five categories of proportions were predefined in the survey. 
microbiological monitoring and potentially lower transmission/acquisition risks may become evident in the 2021 Cystic Fibrosis Foundation Patient Registry data.

In conclusion, we found rapid uptake of video/telehealth, yet the centres we surveyed here reported challenges with uptake and quality of home spirometry, in contrast to prior studies [3, 4]. These centres also reported more challenges with microbiological monitoring via telehealth compared to spirometry (open comments and figure 1). Based on the comments that several centres only obtained cultures from expectorating patients, we speculate that microbiology assessment may become more difficult with decreasing numbers of patients who can expectorate. Our findings also highlight the need to develop or enhance methods for collecting respiratory cultures at home; for example, during airway clearance, and to use clinic visits for home spirometry teaching. Reassuringly, the team structure for cystic fibrosis care remained functional and most patients with cystic fibrosis cared for at our study sites were able to access cystic fibrosis-specific care.

Marianne S. Muhlebach $\oplus^{1,2}$, Fiona Cunningham ${ }^{1}$, John J. LiPuma ${ }^{3}$, Juyan J. Zhou ${ }^{4}$ and Lisa Saiman ${ }^{4,5}$

${ }^{1}$ Dept of Pediatrics, Division Pulmonology CB\#7217, University of North Carolina, Chapel Hill, NC, USA ${ }^{2}$ Marsico Lung Institute, Chapel Hill, NC, USA. ${ }^{3}$ Dept of Pediatrics, University of Michigan Medical School, Ann Arbor, MI, USA. ${ }^{4}$ Dept of Pediatrics, Columbia University Irving Medical Center, New York, NY, USA. ${ }^{5}$ Dept of Infection Prevention and Control, NewYork Presbyterian Hospital, New York, NY, USA.

Corresponding author: Marianne S. Muhlebach (Marianne_Muhlebach@med.unc.edu)

Conflict of interest: M.S. Muhlebach reports grants from the Cystic Fibrosis Foundation during the conduct of the study and service in a consulting group for Nabriva Pharmaceuticals outside the submitted work. F. Cunningham has nothing to disclose. J.J. LiPuma has nothing to disclose. J.J. Zhou reports grants from the US Cystic Fibrosis Foundation during the conduct of the study. L. Saiman reports grants from the Cystic Fibrosis Foundation during the conduct of the study.

Support statement: This study was supported by Cystic Fibrosis Foundation grant MUHLEB19A0. Funding information for this article has been deposited with the Crossref Funder Registry.

References

1 Shakkottai A, Kaciroti N, Kasmikha L, et al. Impact of home spirometry on medication adherence among adolescents with cystic fibrosis. Pediatr Pulmonol 2018; 53: 431-436.

2 Mogayzel PJ, Naureckas ET, Robinson KA, et al. Cystic Fibrosis Foundation pulmonary guideline. Pharmacologic approaches to prevention and eradication of initial Pseudomonas aeruginosa infection. Ann Am Thorac Soc 2014; 11: 1640-1650.

3 Lechtzin N, Mayer-Hamblett N, West NE, et al. Home monitoring of patients with cystic fibrosis to identify and treat acute pulmonary exacerbations. elCE study results. Am J Respir Crit Care Med 2017; 196: 1144-1151.

4 Logie K, Welsh L, Ranganathan SC. Telehealth spirometry for children with cystic fibrosis. Arch Dis Child 2020; 105: 1203-1205. 